\title{
Impacto en la Producción Científica de un Programa Experiencial de Preparación para la Investigación Dirigido a Docentes Universitarios
}

\author{
Santiago F. Barriga ${ }^{(1) *}$, Olga G. Barbón ${ }^{(1)}$, Carlos V. Buenaño(2), Luis F. Barriga ${ }^{(1)}$ \\ (1) Universidad Nacional de Chimborazo (UNACH), Ave 10 de agosto y Eloy Alfaro, Riobamba, Ecuador. \\ (e-mail: sbarriga@unach.edu.ec olgagloria29@yahoo.com) \\ (2) Escuela Superior Politécnica de Chimborazo (ESPOCH), Panamericana Sur km 11/2, Riobamba, Ecuador.
}

${ }^{*}$ Autor a quien debe ser dirigida la correspondencia

Recibido Ago. 28, 2017; Aceptado Nov. 6, 2017; Versión final Dic. 13, 2017, Publicado Jun. 2018

\begin{abstract}
Resumen
Se analizan los cambios en la producción científica, en un grupo de docentes universitarios, tras participar en un Programa Experiencial de Preparación para la Investigación. Entre abril y septiembre del 2017, se llevó a cabo un estudio cuantitativo, descriptivo y cuasi-experimental en universidades de la zona 3 del Ecuador. Se realizaron varias medidas: un pretest antes de la implementación del programa, un postest(1) justo al finalizar el primer corte de la actividad formativa y un postest(2) a los seis meses de iniciado el programa, mediante la aplicación del mismo cuestionario a ambos grupos. Entre los resultados más relevantes se obtuvo que mientras que en el grupo control no hubo cambios, en el grupo experimental se aumentó la producción de ponencias, de artículos científicos de revisión, de artículos originales y la publicación de artículos en revistas indexadas de forma significativa en el postest(1).
\end{abstract}

\section{Impact on the Scientific Production of a Preparation for Research Experiential Program for University Teachers}

\begin{abstract}
Changes in scientific production in a group of university professors after participating in a Preparation for Research Program are analyzed. Between April and September 2017, a quantitative, descriptive and quasiexperimental study was carried out at universities belonging to zone 3 of Ecuador. Several measures were taken: a pretest before the program was implemented, a postest (1) just at the end of the first cut of the training activity and a postest (2) six months after the program was started, applying the same questionnaire to both groups. Among the most relevant results was found that while in the control group there were no changes, the experimental group increased the production of papers, scientific review papers, original papers and articles published in indexed journals significantly in posttest(1).
\end{abstract}




\section{INTRODUCCIÓN}

En términos generales, los últimos tiempos han representado un impulso significativo para la investigación universitaria, ello ha conducido a que, amparada en la propia misión eminentemente social de las universidades, la producción científica de los docentes universitarios se convierta en un instrumento herramienta idóneo para el continuo mejoramiento de la calidad académica en las Instituciones de Educación Superior (IES) a nivel global, con lo cual resulta cada vez más necesario que su producción científica y los factores que intervienen en la misma sean objeto de reflexión y de análisis (García de Fanelli, 2014). Ahora bien, la producción científica del profesorado universitario es el resultado del entrecruce de capacidades, prácticas e intencionalidades diversas que surgen del entramado de tres aspectos fundamentalmente: i) Las políticas de investigación científica llevadas a cabo por las administraciones de la ciencia y la tecnología y su capacidad para generar propuestas de desarrollo científico y promover y potenciar las actividades de transferencia de conocimiento e incentivar la investigación educativa y la aplicación del método científico en las aulas universitarias; ii) Los niveles de calidad y los grados de sistematización de las prácticas y experiencias concretas de formación desarrolladas en cada enclave académico y la capacidad de estas para armonizar y coordinar las diferentes competencias en materia de investigación científica, así como para expandir la producción científica docente; y iii) La innovación, los aportes de los docentes y la capacidad de estos para contribuir al desarrollo teórico y práctico de las distintas áreas del conocimiento. (Montoya, 2015)

Aunque en la arena internacional tradicionalmente, la investigación ha ocupado un lugar privilegiado en las universidades y en ellas los profesores han jugado un rol protagónico en la producción de conocimiento, más recientemente la universidad ecuatoriana tiene como uno de sus postulados más preciados la producción científica de los docentes producto de su participación en actividades de investigación, las cuales han venido a incorporarse como parte consustancial de su labor diaria. (Ley Orgánica de Educación Superior LOES, 2010). Por tanto, en las condiciones actuales, la necesidad de incrementar la producción científica de los docentes universitarios ecuatorianos ha promovido la creación y el desarrollo de pequeños grupos de investigadores, con lo cual puede afirmarse que dicha necesidad se encuentra indisolublemente ligada al desarrollo de competencias y de habilidades investigativas en estos sujetos, cuya formación permanente, de cara a las exigencias que surgen a partir de la revalorización de la universidad como centro de producción de conocimientos, amerita ser estudiada en profundidad.

Paradójicamente, aunque los resultados proporcionados por investigaciones recientes sobre la evaluación del impacto de la capacitación en la producción científica de los docentes reconocen que la misma constituye una necesidad estratégica, como vía para el desarrollo de una universidad (Nicol, Thomson y Breslin, 2014; Wiek, Xiong, Brundiers, y Leeuw, 2014), de su política científica (Parra, 2007; Gregor, y Hevner, 2013; ), así como de su gestión en función de profesionalización de los docentes que laboran en ella (Barbón, López y Figueredo, 2014), además de orientar la toma de decisiones acertadas al momento de elaborar políticas de investigación (Campos, 2015; Bornmann, 2013; Bozeman, Fay, y Slade, 2013), el impacto científico real de las capacitaciones en este ámbito, no ha sido objeto de análisis de manera sistemática por parte de los investigadores, por lo que se obstaculiza la evaluación objetiva del efecto logrado con las diversas alternativas de formación permanente al no existir criterios fiables que permitan obtener certezas científicas de su impacto.

Sobre este aspecto, autores como Álvarez y Juncosa (2014); Ricardo y Velástegui (2015). y Barbón et. al. (2017) han argumentado sobre la pertinencia, la viabilidad y la importancia de que la formación permanente en investigación provoque los cambios necesarios en los resultados científicos de los docentes universitarios del Ecuador, de manera que cumplan con su función social y no se limiten a su difusión en el seno de los centros académicos. Esto significa prestar atención a la urgencia de revisar la formación permanente que reciben estos docentes en materia de investigación. En tal sentido, es clara la necesidad de que esta formación se enfoque en mejorar las habilidades investigativas del profesorado, y para ello se requiere desarrollar investigaciones centradas en superar la ausencia de datos concretos sobre el impacto científico real de esta formación en algunos contextos que permitan; en primer lugar realizar análisis sobre sus potencialidades, sus retos y su verdadera utilidad; en segundo lugar que aporten a la comprensión y transformación cualitativa de este fenómeno en la práctica y, por último, que contribuyan a diseñar políticas de respaldo a la implementación de programas y planes de formación permanente que se correspondan cada vez más con las necesidades de estos sujetos.

Con base en lo hasta aquí descrito, el presente trabajo se centra en analizar los cambios en la producción científica, en un grupo de docentes universitarios, tras participar en un Programa Experiencial de Preparación para la Investigación. Los datos obtenidos (de los cuales solo se reportan aquí resultados parciales), dan cuenta de hasta qué punto la producción científica de los docentes se ha visto modificada por este novedoso programa. 


\section{METODOLOGÍA}

Durante el año 2017, se llevó a cabo un estudio cuantitativo, descriptivo y cuasi-experimental en universidades de la zona 3 del Ecuador. Para aplicar el Programa Experiencial de Preparación para la Investigación, y establecer los contrastes se trabajó con un grupo control, conformado por 25 docentes de la Universidad Regional Autónoma de los Andes (UNIANDES). Se incluyó un grupo experimental de 25 docentes de la Universidad Nacional de Chimborazo (UNACH), en ambos grupos los sujetos integrantes no fueron asignados al azar. Entre abril y septiembre del 2017, se realizaron varias medidas: un pretest antes de la implementación del programa, un postest(1) justo al finalizar el primer corte de la actividad formativa y un postest(2) a los seis meses de iniciado el programa, mediante la aplicación del mismo cuestionario a ambos grupos. Las hipótesis planteadas para esta investigación son: 1) No existirán diferencias significativas de medias en el pretest, según las puntuaciones de las dimensiones analizadas, entre los sujetos del grupo experimental y los del grupo de control; y 2) A partir de la implementación del Programa Experiencial de Preparación para la Investigación se producirán diferencias significativas de medias en el primer postest (postest1) entre los sujetos de los grupos experimentales, respecto al pretest. Se cree que no habrá diferencias entre las medidas de los grupos control respecto a las medidas pretest.

El Programa Experiencial de Preparación para la Investigación, desarrollado con el grupo experimental, tuvo una duración de 10 horas presenciales distribuidas en 5 encuentros y 60 horas no presenciales. La denominación de experiencial se debe a que en el mismo se privilegió el intercambio de experiencias prácticas de investigación, para lo cual se utilizaron técnicas vivenciales (animación, análisis y reflexión grupal). Las temáticas impartidas fueron solicitadas por los participantes, quienes además podían elegir a los facilitadores para cada uno de los encuentros. Los cuales se hallaban caracterizados por el uso de las TICs, de las redes sociales académicas o científicas y de las metodologías activas. Las temáticas se programaron empleando distintas estrategias docentes; tales como: conferencias, seminarios, y actividades prácticas de trabajo en grupo. Se llevó a cabo por dos docentes en cada encuentro, cinco de ellos profesores titulados con Doctorados afines a las ciencias de la educación, y dos son revisores y/o editores de revistas científicas indexadas. El programa quedó estructurado en las siguientes unidades temáticas:

I: Introducción a la publicación científica. Experiencias y lecciones aprendidas.

II: Metodología y trabajo de campo arqueológico para su difusión.

III: Alternativa para implementar un sistema de ciencia e innovación en la Facultad y Cirugía abierta a un artículo científico.

IV: Modelos pedagógicos y su aplicación tecnológica en los nuevos ambientes de aprendizaje.

V: Formulación de Proyectos utilizando la Metodología de Marco Lógico.

El grupo control, simultáneamente, recibió un Programa Experiencial de Preparación para la Investigación, en el cual docentes del mismo nivel y experiencia similar a aquellos que trabajaron con el grupo experimental, emplearon las TICs y las mismas metodologías activas de enseñanza. Sin embargo, este grupo no tuvo acceso al intercambio de experiencias prácticas de investigación con los docentes expositores, ni se les facilitó que pudieran seleccionarles a estos o a las temáticas de estudio.

En el primer encuentro se pasó un cuestionario online de manera individual y autoadministrada. Previo a su aplicación, a los docentes de ambos grupos se les indicó que el objetivo del estudio era conocer el impacto de algunos aspectos en la producción científica de los docentes con el propósito de atenuar el sesgo de selección forzada por categorías insuficientes y considerando que, si bien se preservó el anonimato, los cuestionarios incluían un código para identificar que pertenecían al mismo docente, 3 meses después, se volvió a aplicar el cuestionario, finalizada la actividad, a los 6 meses, se aplicó por última vez para comprobar la modificación en las variables estudiadas. El cuestionario constaba de una breve introducción inicial, una sección para el registro de los datos sociodemográficos y de identificación y de clasificación de la muestra. Además se estructuró en 7 ítems organizados, atendiendo a las variables estudiadas: Producción de capítulos de libros; Producción de artículos científicos originales; Producción científicos de revisión; Producción de ponencias y Producción de artículos científicos en revistas indexadas. Las respuestas a los ítems se realizaron en escalas cuantitativas. A cada encuestado se le informó que su participación en el estudio era voluntaria y anónima y que los resultados de la misma no iban a ser utilizados con fines ajenos a esta investigación.

Los datos obtenidos fueron almacenados en una base de datos de Microsoft Excel 2010, confeccionada al efecto, y se empleó el paquete estadístico SPSS en su versión 21.0 para Windows. Se calcularon los estadísticos descriptivos, media $(M)$ y desviación típica $(D E)$ para cada uno de los ítems. Se empleó el análisis de Kolmogorov-Smirnov para confirmar la normalidad en la distribución de la muestra $(p=0,07)$ y se estudió la fiabilidad de las dimensiones propuestas a través del índice de consistencia interna alfa de Cronbach. A fin de conocer si existían diferencias estadísticamente significativas en las variables entre los dos grupos antes de la intervención, preliminarmente, con los datos recogidos en el pretest (experimental vs control), se realizó 
la prueba $T$ de Student para muestras independientes. (Tabla 1). Se analizaron las diferencias entre grupos en pre, postest1 y postest2 teniendo en cuenta la puntuación de ganancia (Best, 1970). (Tabla 2) Para ello se calculó la diferencia de cada sujeto entre las puntuaciones postest1 y pretest, analizando a continuación las diferencias inter-grupo mediante la prueba $T$ de Student para muestras relacionadas. De igual manera se calcularon las diferencias transcurridos seis meses de la intervención, aunque, en este caso, se tuvieron en cuenta las puntuaciones obtenidas en el postest2 y el postest1 de ambos grupos. (Tabla 2) Se realizó también un análisis de correlaciones canónicas.

\section{RESULTADOS}

Se presentan y discuten los principales resultados del estudio preliminar realizado y de los efectos de la intervención a corto y a mediano plazo.

\section{Análisis preliminar}

Para comprobar que se trataba de dos grupos homogéneos, se analizaron las diferencias entre grupos mediante la Prueba T para muestras independientes. En la Tabla 1 se aprecian las medias y las desviaciones estándar, así como el resultado de la Prueba T correspondientes al pretest, postest1 y postest2.

La primera hipótesis planteada fue que no existirían diferencias significativas en las medias del pretest. Los datos muestran que no existieron diferencias significativas en las variables analizadas, por tanto se corroboró que estábamos en presencia de grupos homogéneos desde el inicio del estudio: Producción de capítulos de libros $(t=0.884 ; p=0.381)$, producción de artículos científicos originales $(t=1.375 ; p=0.176)$, producción de artículos científicos de revisión ( $t=1.178 ; p=0.224)$, producción de ponencias $(t=1.867 ; p=0.068)$ y publicación de artículos originales en revistas indexadas $(t=1.414 ; p=0.164)$. Esto significa que ambos grupos eran iguales al realizar el pretest, tal como se necesitaba al iniciar la investigación.

Tabla 1: Comportamiento de la Media (M) y desviación típica (DT) de pretest, postest1, postest2 en grupo experimental y grupo control $\left({ }^{*} \mathrm{p}<0.05\right)$

\begin{tabular}{|c|c|c|c|c|c|}
\hline \multirow{2}{*}{ Variables dependientes } & & \multicolumn{2}{|c|}{ Grupo experimental } & \multicolumn{2}{|c|}{ Grupo control } \\
\hline & & $\mathrm{M}$ & $\mathrm{DE}$ & $\mathrm{M}$ & $\mathrm{DE}$ \\
\hline \multirow[t]{3}{*}{ Producción de capítulos de libros } & \multirow{3}{*}{$\begin{array}{l}\text { Pretest } \\
\text { Postest1 } \\
\text { Postest2 }\end{array}$} & 0.44 & 1.00 & 0.39 & 0.52 \\
\hline & & 0.54 & 0.68 & 0.44 & 0.82 \\
\hline & & 0.52 & 0.96 & 0.40 & 0.86 \\
\hline \multirow[t]{3}{*}{ Producción de artículos científicos originales } & \multirow{3}{*}{$\begin{array}{l}\text { Pretest } \\
\text { Postest1 } \\
\text { Postest2 }\end{array}$} & 1.44 & 1.58 & 1.35 & 0.73 \\
\hline & & $1.80^{*}$ & 1.41 & 1.47 & 1.26 \\
\hline & & 1.78 & 1.68 & 1.34 & 0.94 \\
\hline \multirow[t]{3}{*}{ Producción de artículos científicos de revisión } & \multirow{3}{*}{$\begin{array}{l}\text { Pretest } \\
\text { Postest1 } \\
\text { Postest2 }\end{array}$} & 1.56 & 1.85 & 1.48 & 0.59 \\
\hline & & $1.96^{*}$ & 1.45 & 1.52 & 1.32 \\
\hline & & 1.86 & 1.56 & 1.45 & 1.24 \\
\hline \multirow[t]{3}{*}{ Producción de ponencias } & \multirow{3}{*}{$\begin{array}{l}\text { Pretest } \\
\text { Postest1 } \\
\text { Postest2 }\end{array}$} & 2.52 & 1.29 & 2.41 & 1.28 \\
\hline & & $2.96^{*}$ & 1.31 & 2.46 & 1.32 \\
\hline & & 2.76 & 1.44 & 2.40 & 1.35 \\
\hline \multirow[t]{3}{*}{ Artículos científicos en revistas indexadas } & \multirow{3}{*}{$\begin{array}{l}\text { Pretest1 } \\
\text { Postest1 } \\
\text { Postest2 }\end{array}$} & 0.86 & 1.22 & 0.76 & 0.41 \\
\hline & & $1.12^{*}$ & 1.66 & 0.81 & 0.56 \\
\hline & & 1.11 & 1.68 & 0.73 & 0.56 \\
\hline
\end{tabular}

\section{Efectos de la intervención}

La tabla 2 muestra los resultados de las medias y desviaciones estándar de los grupos experimental y de control. Al comparar los resultados alcanzados por el grupo experimental y el grupo control la prueba de T arroja diferencias significativas intra-grupos entre las medias de las variables estudiadas. En el grupo control no se encontraron diferencias significativas. Sin embargo, en el grupo experimental las máximas diferencias se hallaron en la producción de ponencias ( $\mathrm{t}=2.68 ; \mathrm{p}=0.02)$, seguida de la producción de artículos científicos de revisión ( $t=2.34 ; p=0.02)$. Le siguen la producción de artículos originales $(t=2.17 ; p=0.03)$ y la producción de artículos en revistas indexadas $(\mathrm{t}=2.05 ; \mathrm{p}=0.04)$. Para la variable producción de capítulos de libros las diferencias no fueron significativas. Esto evidenció que mientras que en el grupo control no hubo cambios, en el grupo experimental se aumentó la producción de ponencias, de artículos científicos de revisión, de artículos originales y de artículos en revistas indexadas de forma significativa en el postest1. Se ha cumplido la segunda hipótesis y se puede afirmar que el programa ha sido eficaz. Puede observarse que las diferencias entre el postest1 y el postest2 no fueron significativas para ninguno de los dos grupos; las medias se mantuvieron casi iguales. Fueron menores las diferencias en el grupo experimental que en el de control. 
Tabla 2: Diferencias entre grupo experimental y grupo control en pretest, postest1 y postest2 según la puntuación de ganancia y la Prueba T para muestras relacionadas $\left({ }^{*} \mathrm{p}<0.05\right)$.

\begin{tabular}{|c|c|c|c|c|c|c|}
\hline \multirow{2}{*}{ Variables dependientes } & \multicolumn{3}{|c|}{ Grupo experimental } & \multicolumn{3}{|c|}{ Grupo control } \\
\hline & $\mathrm{M}$ & $\mathrm{DE}$ & Ganancia & $\mathrm{M}$ & $\mathrm{DE}$ & Ganancia \\
\hline $\begin{array}{l}\text { Producción de capítulos de libros } \\
\text { Pretest }\end{array}$ & 0.44 & 1.00 & & 0.39 & 0.52 & \\
\hline Postest1 & 0.54 & 0.68 & 0.10 & 0.44 & 0.82 & 0.05 \\
\hline Postest2 & 0.52 & 0.96 & -0.02 & 0.40 & 0.86 & -0.04 \\
\hline $\begin{array}{l}\text { Producción de artículos } \\
\text { Pretest } \\
\text { científicos originales } \\
\text { Postest } 1\end{array}$ & $\begin{array}{l}1.44 \\
1.80^{\star}\end{array}$ & $\begin{array}{l}1.58 \\
1.41\end{array}$ & 0.36 & $\begin{array}{l}1.35 \\
1.47\end{array}$ & $\begin{array}{l}0.73 \\
1.26\end{array}$ & 0.12 \\
\hline Postest2 & 1.78 & 1.68 & -0.02 & 1.34 & 0.94 & -0.13 \\
\hline $\begin{array}{l}\text { Producción de artículos } \\
\text { Pretest } \\
\text { científicos de revisión } \\
\text { Postest } 1\end{array}$ & $\begin{array}{l}1.56 \\
1.96^{\star}\end{array}$ & $\begin{array}{l}1.85 \\
1.45\end{array}$ & 0.40 & $\begin{array}{l}1.48 \\
1.52\end{array}$ & $\begin{array}{l}0.59 \\
1.32\end{array}$ & 0.04 \\
\hline Postest2 & 1.86 & 1.56 & -0.10 & 1.45 & 1.24 & -0.07 \\
\hline $\begin{array}{l}\text { Producción de ponencias } \\
\text { Pretest }\end{array}$ & 2.52 & 1.29 & & 2.41 & 1.28 & \\
\hline Postest1 & $2.96^{*}$ & 1.31 & 0.44 & 2.46 & 1.32 & 0.05 \\
\hline Postest2 & 2.76 & 1.44 & -0.08 & 2.40 & 1.35 & -0.04 \\
\hline $\begin{array}{l}\text { Artículos científicos } \\
\text { Pretest } \\
\text { en revistas indexadas } \\
\text { Postest1 }\end{array}$ & $\begin{array}{l}0.86 \\
1.12^{\star}\end{array}$ & $\begin{array}{l}1.22 \\
1.66\end{array}$ & 0.26 & $\begin{array}{l}0.76 \\
0.81\end{array}$ & $\begin{array}{l}0.41 \\
0.46\end{array}$ & 0.05 \\
\hline Postest2 & 1.11 & 1.68 & -0.01 & 0.73 & 0.56 & -0.08 \\
\hline
\end{tabular}

Una vez concluida la intervención, teniendo como referencia el grupo experimental y para confirmar la existencia de relaciones entre las variables dependientes estudiadas, se realizó un análisis de correlaciones bivariadas en los resultados del pretest (tabla 3). Se observa una correlación positiva significativa entre la producción de artículos científicos originales y la producción de artículos científicos en revistas indexadas en el pretest. En el postest1 aumentó la significación existente de las correlaciones del pretest y además apareció significación en la relación de la producción de artículos científicos originales con la producción de artículos científicos de revisión y la producción de ponencias. Esto demuestra que con la intervención, al aumentar la producción de artículos científicos originales aumentó la producción de artículos científicos de revisión, de ponencias y de artículos científicos en revistas indexadas; también al acrecentarse la producción de artículos científicos originales se incrementó la producción de ponencias y de artículos científicos en revistas indexadas. Finalmente, con el incremento de la producción de ponencias se acrecentó también la producción de artículos científicos en revistas indexadas.

Tabla 3: Correlación entre las variables estudiadas en el grupo experimental en el pretest y postest 1 . ${ }^{*} p<0.05 ;{ }^{* *} p<0.01$. Nota: en la diagonal superior se indican las correlaciones del pretest y en la diagonal inferior las del postest1

\begin{tabular}{|c|c|c|c|c|c|}
\hline Variables & I & II & III & IV & $V$ \\
\hline I. Producción de capítulos de libros & & 0.14 & 0.17 & 0.11 & 0.10 \\
\hline II. Producción de artículos científicos originales & 0.27 & & 0.16 & $0.483^{*}$ & $0.460^{*}$ \\
\hline III. Producción científicos de revisión & 0.28 & $0.52^{\star *}$ & & & \\
\hline IV. Producción de ponencias & 0.11 & $0.51^{* *}$ & $0.65^{\star *}$ & & \\
\hline V. Producción de artículos científicos en revistas indexadas & 0.25 & $0.72^{* *}$ & $0.69^{* *}$ & $0.71^{\star \star}$ & \\
\hline
\end{tabular}

\section{Impacto a mediano plazo de la intervención}

Una vez trascurridos seis meses desde el programa, se compararon los resultados obtenidos al finalizar la intervención (postest1), con los resultados obtenidos tras los meses indicados con respecto a los mismos 25 docentes del grupo experimental. Se llevó a cabo un segundo postest para comprobar los resultados analizados en las diferentes variables estudiadas. Aquí la puntuación de ganancia se halló restando los resultados del postest1 a los del postest2. En la tabla 2 se observa que la prueba T no mostró resultados significativos, lo que indica que los docentes han mantenido la producción científica durante el paso del tiempo, aún después de concluida la intervención, lo cual es un aspecto positivo que habla a favor de su impacto, no solo a corto, sino también a mediano plazo. 


\section{DISCUSIÓN}

Actualmente el desempeño de las universidades se presenta mediante rankings que las clasifican universidades en función de su producción científica (Machorro y Romero, 2017), unido a la fuerte presión que ello supone han ocurrido transformaciones en el proceso académico científico que han provocado profundos cambios en la identidad de la institución universitaria y sus docentes. Este cambio radical de la identidad de la universidad promueve continuamente un aumento en el trabajo productivo intangible del profesor. La producción científica nace de la condición de científico de cada investigador, su empoderamiento del conocimiento y su reflexión crítica en un campo de estudio que hace más eficaz la pertinencia de las IES frente a las necesidades de la sociedad. Tras analizar la producción científica en un grupo de docentes universitarios después de participar en un Programa Experiencial de Preparación para la Investigación, se entiende efectivamente como un instrumento del cambio sustantivo para la redacción científica en el personal académico.

Además, la producción científica es en potencia un trabajo colaborativo que se desarrolla con redes y grupos de investigación y cuya función se basa en la capacitación permanente. De hecho, la importancia concedida a la producción científica del personal docente pone de manifiesto hasta qué punto las exigencias actuales para revalorizar a la universidad tienen relación con el mejoramiento de la calidad de la preparación para el desarrollo de la producción científica. El enfoque metodológico adoptado en este estudio resultó apropiado para cumplir el objetivo propuesto, la implementación del Programa Experiencial de Preparación para la Investigación tenía la intención de establecer aquellas diferencias significativas en los resultados obtenidos con la aplicación de este programa, basado en el intercambio de experiencias sobre investigación, el uso de las TICs y metodologías activas.

Amén de que se trata de un estudio cuasi-experimental, lo que implica que las conclusiones respecto de la eficacia del programa de intervención deben ser cuidadosas, la investigación realizada consiguió establecer la diferencia entre el grupo de control y el grupo experimental, después de una evaluación inicial, sobre la producción científica alcanzada en los últimos cuatro años a partir de las variables planteadas: producción de capítulos de libros, producción de artículos científicos originales, producción de artículos científicos de revisión, producción de ponencias y publicación de artículos originales en revistas indexadas. Por otra parte, de acuerdo con los datos obtenidos en la prueba de T, la idoneidad del programa de preparación aplicado a los sujetos experimentales permitió establecer datos coherentes y la cuantificación significativa de las prácticas relacionadas con la producción científica.

El estudio resaltó de forma significativa una mayor producción de ponencias con un valor de 0.51 equivalente a p $<0.05$, en vista de que existía mayor oportunidad para su aceptación por los pares académicos que integran los comités evaluadores en eventos científicos, que mayormente solicitan exclusivamente la presentación previa del resumen de la ponencia a los participantes, previo a su inscripción, a diferencia del proceso de aceptación de las revistas indexadas, que -mostrando un nivel superior de rigurosidad- requieren de la revisión por pares del manuscrito en su totalidad, como también de otras funciones editoriales para garantizar la calidad de los artículos publicados. Otro hallazgo importante de la investigación es que la misma permite visualizar la producción de artículos científicos de revisión, con un incremento del 0.52 equivalente a $p<0.01$ en el grupo experimental, después de la aplicación del postest. Con lo anterior se evidencia que la intervención propició el desarrollo de las habilidades de búsqueda, revisión y análisis bibliográfico, pues solo así se logra el abordaje crítico y profundo de determinada temática desde otra perspectiva, que requiere la revisión bibliográfica, como modalidad de publicación (Chacón, Herrera y Villabona, 2013), de gran utilidad para la organización, categorización y difusión del conocimiento y que desempeña un papel preponderante en la transferencia de información entre académicos, científicos, especialistas y sus pares.

Sobre la eficacia de la intervención, esta quedó avalada por los resultados. Mientras que en el grupo control no hubo cambios, en el grupo experimental se aumentó la producción de ponencias, de artículos científicos de revisión, de artículos originales y de artículos en revistas indexadas de forma significativa en el postest1. Este incremento es relevante, si se tiene en cuenta que del total de artículos publicados en revistas indexadas de alto impacto en los últimos años, aquellos pertenecientes al área de ciencias sociales a nivel mundial bordean el rango del 10\% (Brembs, et. al, 2013). En trabajos de publicación más reciente, se encuentran algunos que en forma directa o indirecta corroboran esta idea. En un estudio realizado por Barbón et. al (2017) se muestra que, en el medio académico, es común encontrar que la redacción se convierte en un problema para el investigador, cuando este intenta publicar sus trabajos en medios especializados. La dificultad que entraña el ejercicio de poner por escrito eficientemente lo que se ha investigado, en ocasiones, frustra al investigador y retrasa la difusión del conocimiento. (Hayter y Feeney, 2016) A ello pudiera agregarse que los docentes de las ciencias sociales deben añadir a esa dificultad, otra aún mayor: la escasa representación de las revistas en español de esta área del conocimiento en las bases de datos de Scopus o Web of Science. Por ello, aunque las revistas científicas de corte social que publican en idioma español, están consiguiendo 
hacerse un espacio en estas clasificaciones, se recomienda democratizar aún más el impacto de los manuscritos de estos docentes o investigadores, facilitando el ingreso de dichas revistas a estas reconocidas bases de datos. El hecho de que la popularidad de la ciencia está en la etapa embrionaria en el área de las ciencias sociales, no significa que esta no podría ser más explotada, tal vez sea esta una de las razones por las que las IES en el Ecuador se encuentran aún en franco proceso de acercamiento a las ciencias sin fronteras.

Por otro lado, es pertinente destacar que aunque algunos autores consideran que debido a la dilación inherente al proceso de publicación en revistas indexadas, los docentes recurren a canales alternativos para difundir los resultados de sus investigaciones (Bornmann, 2015), en contraste, ciertos investigadores aseguran que hoy en día resulta más fácil el acceso a las bases de datos de revistas de impacto (Botija y Martínez, 2014) y más factible publicar por la cercanía y el manejo de las nuevas tecnologías (Más-Bleda et. al, 2016), ya que en la actualidad las revistas indexadas emplean estos medios para la recepción de los artículos. Ello pudiera explicar que los docentes del grupo experimental hayan conseguido mantener la producción científica durante el paso del tiempo, aún después de concluida la intervención, lo cual es un aspecto que muestra su efecto generador de hábitos de publicación entre estos docentes.

Por último, este estudio reveló aspectos que deben ser considerados en la organización de programas para la preparación de la investigación, que permitan visualizar un panorama general del valor del evento. En primer lugar, la organización del evento debe considerar en el aspecto experiencial la utilización de las redes sociales académicas o científicas que se desarrollan espontáneamente y que constituyen una de las principales razones de la participación. En este sentido, las interacciones que se desarrollan en las mismas van más allá de la exposición formal en las conferencias, pues los procesos de comunicación informal ocurren naturalmente entre los participantes (Cantón y García, 2015) y estos alcanzan a aprender tanto de las experiencias vividas alrededor del tema de la investigación como de los propios contenidos investigativos que se abordan. De los resultados aquí comentados se derivan, al menos, dos áreas problemáticas sin solucionar: el impulso a las revistas científicas que recogen temas sociales para incorporarlas a la corriente mundial y la humanización de la preparación para la investigación.

\section{CONCLUSIONES}

En resumen, respecto a la eficacia del programa, considerando que se trata de un efecto específico para la muestra analizada, de acuerdo a los resultados de este estudio, y de su discusión y análisis se pueden extraer las siguientes conclusiones: a) El impacto de un Programa Experiencial de Preparación para la Investigación, en el cual se privilegie el intercambio de experiencias prácticas de investigación y de temáticas solicitadas por los participantes, quienes además puedan elegir a los facilitadores- profesores titulados con Doctorados afines a las ciencias de la educación, y dos son revisores y/o editores de revistas científicas indexadas - para cada uno de los encuentros, en los cuales se empleen las TICs, las redes sociales académicas o científicas y las metodologías activas, es positivo; b) No solamente se logró que el grupo experimental incrementara la producción de ponencias, de artículos científicos de revisión, de artículos originales y de artículos en revistas indexadas de forma significativa, sino que además este grupo fue capaz de mantener su producción científica durante el paso del tiempo, lo cual es un aspecto que muestra su efecto generador de hábitos de publicación entre estos docentes y que habla a favor del impacto del programa, no solo a corto, sino también a mediano plazo.

\section{AGRADECIMIENTOS}

Los autores agradecen el apoyo de las universidades de la zona 3 del Ecuador involucradas en el estudio realizado.

\section{REFERENCIAS}

Álvarez, L. y Juncosa, J., La evaluación y acreditación de la educación superior ecuatoriana y la producción editorial. Universitas, XII (21), 163-181. https://goo.gl/H6torR. Acceso: 1 Agosto 2017 (2014)

Barbón, O. G., Calderón, Á. del R., Loza, C. A., Garcés, L. y Fernández, J.W., Algunos problemas de docentes universitarios en la elaboración de artículos científicos. Actualidades Investigativas en Educación, 17(1). https://goo.gl/Hbb3cE. Acceso: 1 Agosto 2017 (2017)

Barbón, O. G., López, C. L. y Figueredo, D., Cinco saberes para la formación de la competencia científico-investigativa con enfoque de profesionalización pedagógica. Revista Cubana de Reumatología, 16(2), 253-258. https://goo.gl/ZJPjWY. Acceso: 1 Agosto 2017 (2014)

Best, J.W., Research in education. New Jersey: Prentice Hall (1970)

Bornmann, L., What is societal impact of research and how can it be assessed? A literature survey. Journal of the Association for Information Science and Technology, 64(2), 217-233. Acceso: 1 Agosto 2017 (2013) 
Bornmann, L., Alternative metrics in scientometrics: A meta-analysis of research into three altmetrics Scientometrics, 103(3), 1123-1144. Acceso: 1 Agosto 2017 (2015)

Botija, M. y Martínez, L., ¿Cómo escribir un artículo científico desde la intervención social? TSnova, 2(10), 71-79 (2014)

Bozeman, B., Fay, D., y Slade, C. P., Research collaboration in universities and academic entrepreneurship: the-state-ofthe-art. The Journal of Technology Transfer, 38(1), 1-67. Acceso: 1 Agosto 2017 (2013)

Brembs, B., Button, K., y Munafò, M., Deep impact: unintended consequences of journal rank. Frontiers in human Neuroscience, 7. Acceso: 1 Agosto 2017 (2013)

Campos Saborío, N., ¿Por qué es importante que el personal docente investigue? Reflexión a partir de datos de una experiencia de investigación etnográfica. Revista Electrónica Actualidades Investigativas en Educación, 15(3), 1-16 (2015)

Cantón, I., y García A., La comunicación en los centros educativos: un estudio de caso. Tendencias Pedagógicas 19(2), 107-130 (2015)

Chacón, J. W. B., Herrera, J. C. B. y Villabona, M. R., Revisión y análisis documental para estado del arte: una propuesta metodológica desde el contexto de la sistematización de experiencias educativas. Investigación Bibliotecológica: Archivonomía, Bibliotecología e Información, 27(61), 83-105 (2013)

García de Fanelli, A.M., Rendimiento académico y abandono universitario: Modelos, resultados y alcances de la producción académica en la Argentina. Revista Argentina de Educación Superior, (8), 9-38 (2014)

Gregor, S., y Hevner, A. R., Positioning and presenting design science research for maximum impact. MIS quarterly, 37(2), acceso: 1 Agosto 2017 (2013)

Hayter, C. S., y Feeney, M. K., Determinants of external patenting behavior among university scientists. Science and Public Policy, 44(1), 111-120, acceso: 1 Agosto 2017 (2016)

Ley Orgánica de Educación Superior (LOES), Consejo de Educación Superior (CES), Ecuador (2010)

Machorro, F. y Romero, M. V., Propuesta de un Instrumento de Autoevaluación del Desempeño Organizacional en Instituciones Públicas de Educación Superior en México. Formación Universitaria, 10(3), 3-10. https://dx.doi.org/10.4067/S071850062017000300002. Acceso: 1 Agosto 2017 (2017)

Más-Bleda, A., Thelwall, M., Kousha, K., y F. Aguillo, I., Successful researchers publicizing research online: An outlink analysis of European highly cited scientists' personal websites. Journal of Documentation, 70(1), 148-172. Acceso: 1 Agosto 2017 (2014)

Montoya, M. S. R., Acceso abierto y su repercusión en la Sociedad del Conocimiento: Reflexiones de casos prácticos en Latinoamérica/Open Access and its impact on the Knowledge Society: Latin American Case Studies Insights. Education in the Knowledge Society, 16(1), 103 (2015)

Nicol, D., Thomson, A., y Breslin, C., Rethinking feedback practices in higher education: a peer review perspective. Assessment \& Evaluation in Higher Education, 39(1), 102-122. http://dx.doi.org/10.1080/02602938.2013.795518. Acceso: 1 Agosto 2017 (2014)

Parra, M.C., Las políticas de ciencia y tecnología en Venezuela y su Impacto en el sistema universitario en el estado Zulia. Interciencia, 419-426. Recuperado de: https://goo.gl/2fBbbj. Acceso: 1 Agosto 2017 (2007)

Ricardo, M. y Velástegui, M.E., Investigación y vinculación: por el camino a una integración necesaria en la universidad ecuatoriana. UNIANDES EPISTEME: Revista de Ciencia, Tecnología e Innovación, 2 (2), http://186.46.158.26/ojs/index.php/EPISTEME/article/view/100, acceso: 1 Agosto 2017 (2015)

Wiek, A., Xiong, A., Brundiers, K., y van der Leeuw, S., Integrating problem-and project-based learning into sustainability programs: A case study on the School of Sustainability at Arizona State University. International Journal of Sustainability in Higher Education, 15(4), 431-449. http://186.46.158.26/ojs/index.php/EPISTEME/article/viewFile/100/103. Acceso: 1 Agosto 2017 (2014) 